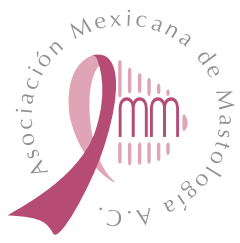

doi: $10.35366 / 99159$

\title{
Semblanza del Dr. Carlos Sánchez Basurto: fundador de la Asociación Mexicana de Mastología
}

\author{
Semblance of Dr. Carlos Sánchez Basurto: founder of the Mexican Association of Mastology \\ Ernesto R Sánchez Forgach*
}

* Cirujano Oncólogo. Ex Presidente de la Asociación Mexicana de Mastología. Consejo Mexicano de Oncología. Miembro de la Asociación Médica Centro Médico ABC. México.
Correspondencia: Dr. Ernesto R Sánchez Forgach Centro Médico ABC Observatorio, Torre Central, $2^{\circ}$ piso, Consultorio 18. Sur 136 Núm. 116, Col. Las Américas, 01120, Alcaldía Álvaro Obregón, Ciudad de México, México. E-mail: mastologica1@gmail. com

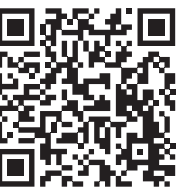

Carlos Sánchez Basurto nació en la Ciudad de México el 7 de diciembre de 1929, hijo de María Luisa Basurto Ramos y Carlos Sánchez Loaeza, realizó su educación básica, secundaria y preparatoria en diversos colegios maristas, incluyendo el Centro Universitario México.

Estudió la carrera de Médico Cirujano en la Facultad de Medicina de la UNAM, realizó su servicio social en Trincheras, Sonora, y se recibió el 31 de julio de 1954.

Médico del Honorable Cuerpo de Bomberos, llevó a cabo la especialidad de Cirugía General en el Hospital General de México de 1954 a 1957. Ese mismo año se incorporó al Pabellón 13 de Oncología de dicha institución, bajo la tutela de los maestros Horacio Zalce y José Manuel Velasco Arce, donde llegó a ser médico adscrito por oposición. Animado por su amigo, el Dr. José Noriega Limón, realizó una estancia médica en el MD Anderson Cancer Center en Houston, Texas, entre 1958 y 1959, bajo la tutela de Murray Copeland.

Después, ingresó a la Unidad de Oncología del IMSS en la calle de Niños Héroes, donde se desempeñó como Jefe de Residentes y posteriormente formó parte de la primera generación de médicos del Hospital de Oncología del Centro Médico Nacional en donde, desde su inauguración, ocupó la jefatura de los servicios de Tumores Mixtos, de Cabeza y Cuello, y de Tumores Mamarios entre 1962 y 1980.

Invitado por Luis Castelazo Ayala, Carlos MacGregor y Javier Soberón Acevedo, fue fundador del Hospital de México (actualmente Ángeles México) y ejerció en esa institución desde 1981 hasta 2003.

En enero de 2004 se cristalizó su sueño de establecer la primera clínica integral en México de enfermedades de la glándula mamaria, el Centro de Estudios Mastológicos conocido como Mastológica Lomas, sitio en el que se realiza el tamizaje, diagnóstico, tratamiento quirúrgico y médico del cáncer de mama.

Posteriormente, abrió Oncológica Lomas, con atención a todos los padecimientos neoplásicos malignos en donde se inició un centro de infusiones y se llevaron a cabo protocolos de investigación.

Por otra parte, fue profesor de las cátedras de «Introducción a la clínica» de la Facultad de Medicina de la UNAM de 1956 a 1958, y de «Patología del Aparato Digestivo» y de «Oncología» en la Escuela Superior de Medicina del Instituto Politécnico Nacional de 1955 a 1985.

También fue profesor titular del pregrado de la cátedra de «Oncología» de la Universidad Anáhuac y adjunto de la misma materia en la Universidad La Salle. Laboró como profesor

Citar como: Sánchez FER. Semblanza del Dr. Carlos Sánchez Basurto: fundador de la Asociación Mexicana de Mastología. Rev Mex Mastol. 2020; 10 (3): 98-100. https://dx.doi.org/10.35366/99159 
asociado del curso universitario de Ginecología del Hospital Ángeles México hasta el año 2020.

Además, asistió y participó como ponente en múltiples cursos nacionales de Oncología, de Cirugía General y Ginecología. Cabe destacar que fue profesor titular de cursos de Mastología y de Oncología Quirúrgica en Colombia, Ecuador y México.

\section{SOCIEDADES MÉDICAS}

Ingresó a la Sociedad Mexicana de Estudios Oncológicos en 1959; en 1965 organizó el Congreso Nacional y fue su presidente en 1970; fue miembro activo hasta el 2020.

Ingresó como fellow del Colegio Americano de Cirujanos en 1985 y fue Presidente del Capítulo Mexicano en 1996. Se incorporó al Colegio Internacional de Cirujanos en 1976, fue Presidente del Capítulo México (1999-2000) y organizó el Congreso Nacional en la ciudad de Puebla, así como el Intercapitular de Norteamérica en Cancún en 1999.

Fue fundador y primer Presidente de la Sociedad y el Colegio Médico del Hospital de México de 1974 a 1976.

Como vicepresidente de la Federación Latinoamericana de Sociedades de Cancerología (FLASCA) realizó los Congresos Integrados de Cancerología en 1975 y fue Secretario de las Jornadas Franco-Mexicanas de Oncología en 1986.

Fue miembro de la Asociación Médica del Hospital ABC desde 1965 y uno de sus decanos hasta el año 2020.

Ingresó a la Academia Mexicana de Cirugía en 1980, de la cual fue Presidente de la Comisión de Convivencias Quirúrgicas en el año 2002 y Académico Emérito hasta el año 2020.

Formó parte del Comité Editorial de la revista «Cirugía y Cirujanos» por varias décadas, así como de las revistas «Mexicana de Mastología», «Oncología mexicana y argentina» y «Ginecología y Obstetricia».

Fue miembro honorario de varias sociedades oncológicas y mastológicas latinoamericanas, acudió a sus congresos y a los de la Sociedad Internacional de Senología (SIS).

De esta forma, dio paso a lo que quizá fue el logro profesional más trascendente de su vida: la fundación de la Asociación Mexicana de Mastología. Con gran interés en reunir a los profesionales de la medicina que tuvieran nexos con el estudio de la glándula mamaria, convocó a diversos especialistas afines y constituyó la primera Mesa Directiva en 1986 integrada por Carlos Sánchez Basurto, Gerardo Vázquez Fritz, Francisco Tenorio González, Roberto Velasco Almeida y Ernesto Sánchez Forgach.

Fue miembro de la Sociedad Internacional de Senología desde 1980 y su presidente en el bienio 2000-2001. Presidente del undécimo Congreso Mundial de Patología Mamaria, Cancún, México, en 2001.

Presidente del Comité Científico de 2002 a 2008 y Presidente de las Sociedades Internacionales de 2007 a 2009.

Como miembro de la Federación Latinoamericana de Mastología (FLAM) acudió a la mayoría de sus congresos.

Fue miembro fundador $y$ activo del Consenso Mexicano para el diagnóstico y tratamiento del cáncer de mama desde su primera reunión en Colima en 1994.

Fue miembro titular de la Asociación Mexicana de Ginecología y Obstetricia desde 1979.

Fundador de la Comisión Nacional de Arbitraje Médico en el año 2000.

Ingresó por invitación al International Society of Surgery en 2001.

Perteneció a la Comisión Nacional de Consejos de Especialistas y a la Asociación Médica de Facultades y Escuelas de Medicina de 2000 a 2002.

\section{PUBLICACIONES}

Autor y coautor en varios artículos de la especialidad en revistas nacionales e internacionales.

En 1986 editó y publicó «Patología mamaria, primer tratado de la especialidad en México». Siguieron los libros «Compendio de patología mamaria», «International Senology», «Tratado de las enfermedades de la glándula mamaria» y más recientemente el libro «Cáncer de mama: actualidades y controversias».

\section{CERTIFICACIONES Y RECONOCIMIENTOS}

Miembro del Consejo Mexicano de Cirugía General entre 1976 y 2004. 
Recibió medalla al mérito en Mastología «Dr. José Manuel Velasco Arce» en 1990.

Consejero fundador del Consejo Nacional de Oncología desde 1996.

Médico distinguido por el gobierno del Distrito Federal en el año 2010.

Diploma de honor «Leader in Senology» de la SIS en el año 2010.

Académico emérito de la Academia Mexicana de Cirugía en 2011.

En 2014 se constituye el Comité de Ética «Carlos Sánchez Basurto».

Maestro de Mastología por la Federación Latinoamericana de Mastología en 2017.

Distinción al mejor oncólogo en la reunión Best of ASCO del año 2018.

Reconocimiento por su trayectoria por la Sociedad Mexicana de Oncología en noviembre de 2018.

Tuvimos el gusto de develar en su presencia el busto que se le dedicó por parte de la Asociación Mexicana de Mastología en el Congreso Nacional realizado en Xalapa, con motivo de su onomástico en noviembre de 2019.

Tuvo tres hermanas: Alicia, Martha y María Elena.

Conoció a Mari Carmen Forgach Marcor en junio de 1959. Contrajeron nupcias el 12 de diciembre de ese mismo año y tuvieron dos hijos: Carlos y Ernesto Sánchez Forgach.

\section{FINAL}

Mi padre emprendió el viaje hacia la eternidad el 4 de julio de 2020.

Fue el mejor maestro y amigo.

Honor a quien honor merece. Una vida llena de trabajo, enseñanza, ética y amor a su carrera como pocos lo han demostrado en su dedicación y desempeño a través de muchas décadas. 